\title{
Perfil eletrocardiográfico de ratos com escoliose induzida por colete de PVC
}

\section{Electrocardiographic profile of rats with induced scoliosis by PVC vest}

Resumo A escoliose é caracterizada por alterações anatomo-fisiológicas importantes. Entre os problemas gerados por essas alterações estão os transtornos estéticos, presentes especialmente em adolescentes. Diversos pesquisadores têm se dedicado ao estudo dos comprometimentos gerados pela escoliose, a maioria dos estudos com animais envolve utilização de modelos invasivos para a indução de escoliose. O objetivo do trabalho foi avaliar se o modelo não invasivo de indução de escoliose em ratos promove modificações na funcionalidade cardíaca. Métodos: Ratos Wistar foram divididos em dois grupos ( $\mathrm{n}=8 / \mathrm{cada})$ : Controle $(\mathrm{C})$, escoliótico (E). Os animais do grupo escoliótico foram submetidos à utilização de um colete de policloreto de vinil (PVC) durante 12 semanas consecutivas para a indução da escoliose. Após o período de 12 semanas, foram avaliados os seguintes parâmetros: peso dos animais, a frequência cardíaca (FC), o ângulo do coração (SÂQRS), o intervalo QRS, a pressão arterial (PA), a força de contração na presença de noradrenalina e o padrão $\mathrm{dP} / \mathrm{dt}+$. A análise estatística foi realizada pelo teste de normalidade (Kolmogorov-Smirnov), ANOVA e post test Tukey $(\mathrm{p}<0,05)$. Quanto aos resultados, os ratos do grupo (E) apresentaram menor peso; não houve diferença na FC entre os grupos; o grupo (E) evidenciou desvio de $11^{\circ}$ do SÂQRS; a duração da onda $\mathrm{R}$ e o intervalo PR não diferiram entre os grupos; a força de contração e o padrão $\mathrm{dP} / \mathrm{dt}+$ não tiveram diferença entre os grupos. Desse modo, os dados sugerem que o modelo não invasivo de indução de escoliose não promove alterações na atividade cardíaca e na pressão arterial.

Palavras-chave: Ratos. Escoliose. Eletrocardiografia. FiSIOTERAPIA.

Alexandre Reis Taveira de SouzA ${ }^{\text {IV }}$

BRUNo FERREIRA GoNÇALVES E SILVA ${ }^{\mathrm{V}}$

${ }^{\mathrm{I}}$ Universidade Metodista de Piracicaba (UNIMEP), Piracicaba/ SP - Brasil.

"Universidade Federal de São Carlos (UFSCAR), São Carlos/SP - Brasil. "IIUniversidade Metodista de Piracicaba (UNIMEP), Piracicaba/ SP - Brasil.

${ }^{\text {IV } U n i v e r s i d a d e ~ E s t a d u a l ~ d e ~ C a m-~}$ pinas (UNICAMP), Campinas/ SP - Brasil.

${ }^{v}$ Universidade Paulista (UNIP),

Campinas/SP - Brasil.

Abstract Scoliosis is characterized by important anatomic-physiological changes. Among the problems generated by these changes are the aesthetic disorders, present especially in adolescents. Several researches have been dedicated to the study of the compromises generated by scoliosis, most animal studies involve the use of invasive models for induction of scoliosis. The aim of this study was to evaluate whether the noninvasive model of induction of scoliosis in rats promotes changes in cardiac function. Methods: Wistar rats were divided into 2 groups $(\mathrm{n}=8 /$ each): Control (C), scoliotic (S). The animals of the scoliotic group were submitted to the use of a vest of polyvinyl chloride (PVC) for 12 consecutive weeks for the induction of scoliosis. After the 12-week period, the following parameters were evaluated: weight of the animals, heart rate (HR), body mass index, Heart angle (SÂQRS), QRS interval, arterial 
pressure (BP), contraction force in the presence of noradrenaline and the $\mathrm{dP} / \mathrm{dt}+$ standard. Statistical analysis was performed by the test of normality (Kolmogorov-Smirnov), ANOVA and post-test Tukey $(\mathrm{p}<0.05)$. As for the results, the rats in group (S) showed lower weight, there was no difference in HR between groups, group (S) showed a deviation of $11^{\circ}$ from SÂQRS; The R-wave duration and the PR interval did not differ between groups, the contraction force and the $\mathrm{dP} / \mathrm{dt}+$ standard did not differ between the groups. Thus, the data suggest that the noninvasive model of scoliosis induction does not promote changes in cardiac activity and in the blood pressure.

Key-words: Rats. Scoliosis. Electrocardiography. Physiotherapy.

\section{INTRODUÇÃO}

O eletrocardiograma é um método efetivo no diagnóstico de alterações cardíacas. Entre as alterações classicamente observadas, encontram-se modificações na amplitude e tempo de duração das ondas ou aumento nos intervalos, que podem estar fundamentados em causas congênitas, anormalidades nos canais de sódio ou potássio, uso de medicamentos, distúrbios metabólicos e anormalidades elétricas ocasionadas por modificações anatomo-fisiológicas. Enquanto o intervalo PR é o espaço do eletrocardiograma que representa a despolarização atrial e o retardo fisiológico do estímulo ao passar pelo nódulo atrioventricular (AV), mostra o tempo de condução atrial; por sua vez, o intervalo QT é a porção do eletrocardiograma que corresponde ao tempo necessário para a completa excitação elétrica e a recuperação dos ventrículos, ou seja, é a duração da atividade elétrica ventricular. ${ }^{1,2}$

$\mathrm{O}$ intervalo QT varia inversamente à frequência cardíaca (FC), quanto maior a FC, tanto menor o QT, e vice-versa. Assim, ele deve ser corrigido em relação à FC, essa correção gera o intervalo QTc, que é usado para a detecção da heterogeneidade da repolarização ventricular, e se caracteriza como marcador da arritmogênese provocada por tempos de repolarização não-homogêneos. ${ }^{3}$

As alterações anatomo-fisiológicas da escoliose incluem modificação estrutural das vértebras e costelas com rotação vertebral no plano transverso, desvio lateral no plano frontal e lordose no plano sagital, o que esteticamente gera transtornos, principalmente em crianças e adolescentes. ${ }^{4}$

As deformidades vertebrais na escoliose estão relacionadas à sua patogênese, que ainda permanece desconhecida. Muitas hipóteses têm sido apresentadas, tais hipóteses estão focalizadas sobre fatores genéticos, esqueléticos, miogênicos, químicos, biomecânicos, neuro-hormonais e neurogênicos. ${ }^{5,6}$

Diversos modelos experimentais de escoliose têm sido utilizados em ratos, há predomínio de métodos invasivos que provocam alteração nos ligamentos vertebrais, na cartilagem intervertebral, osteolatirismo, separação mecânica das vértebras, pinealectomia, além da aplicação de sistemas de fixação das vértebras. ${ }^{7-10}$

Recentemente foi desenvolvida uma metodologia não invasiva para a indução de escoliose em ratos, na qual se utiliza colete de PVC na fase pós-desmame, mantido por 12 semanas e assim há indução de escoliose com curvatura de $36^{\circ} .{ }^{11}$ Desse modo, a proposta deste trabalho é avaliar se esse modelo promove modificações na funcionalidade cardíaca.

\section{MÉTOdos}

Foram utilizados ratos albinos, Wistar adquiridos junto à empresa ANILAB', com 
42 dias de vida, ocasião em que um colete de policloreto de vinil (PVC) foi aplicado por 12 semanas consecutivas. Os ratos foram mantidos no Biotério da UNIMEP, alimentados com ração e água ad libitum, e colocados sob ciclo fotoperiódico de $12 \mathrm{~h}$ claro/escuro, a 22 $\pm 2^{\circ} \mathrm{C}$. O trabalho tem a aprovação segundo parecer do Comitê de Ética em Pesquisa Animal da UNIMEP sob protocolo 09/2012.

Os ratos foram divididos em dois grupos experimentais denominados controle e escoliótico com $n=8$ para cada grupo. Enquanto avaliação inicial, os animais foram pesados em balança de uso laboratorial Filizola ${ }^{\circledR}$ para a avaliação de uma possível modificação na massa, ressalta-se ainda que a ingesta de ração também foi determinada. Para a indução da escoliose, foi utilizado o colete de PVC, constituído por duas peças, uma escapular e outra pélvica, interligadas por um fio metálico que determina a inclinação da coluna e, após a retirada do colete, a coluna apresentou inclinação média de $36^{\circ}$ (figura 1).

Para a avaliação das ondas e intervalos eletrocardiográficos, os ratos foram anestesiados com pentobarbital sódico (40 mg/ $\mathrm{kg} / \mathrm{ip}$ ), baseado no estudo de Kumar et $\mathrm{al}^{12}$, onde foi demonstrado que os barbitúricos não alteram o perfil elétrico cardiovascular. ${ }^{12} \mathrm{~A}$ seguir, eletrodos conectados aos canais do eletrocardiógrafo Heart Ware System interfaciado com um computador foram aplicados ao corpo do animal para registro das ondas nos seguintes padrões (DI, DII, DIII, aVR, aVL e aVF) com velocidade de $50 \mathrm{~mm} /$ segundo (figura 2).

Figura 1 - A) Animal imobilizado com os coletes de policloreto de vinil (escapular e pélvico) utilizado na indução da escoliose e B) imagem radiográfica com demonstração da escoliose após 12 semanas de imobilização.
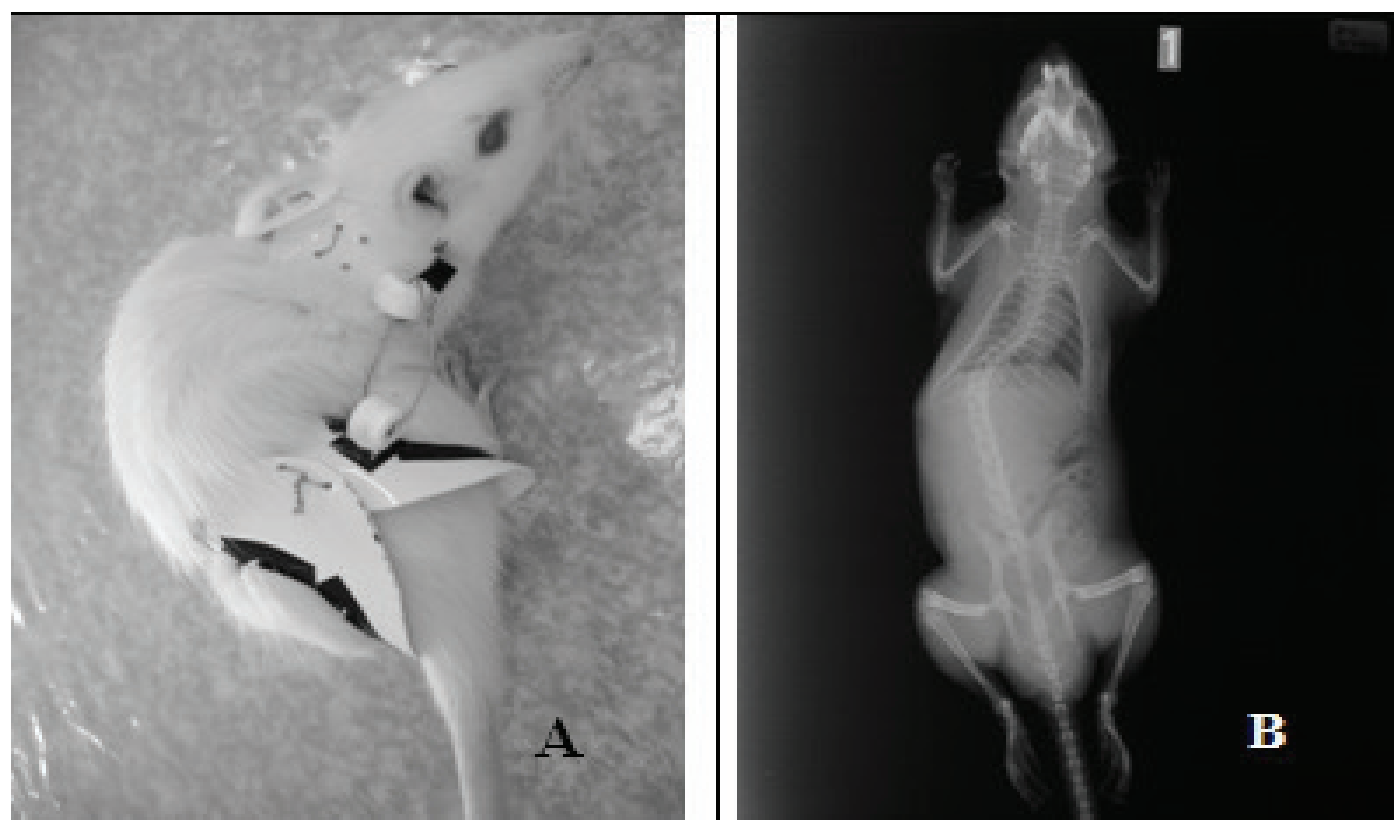
A análise dos registros foi feita por um pesquisador do grupo, devidamente treinado, sendo escolhido na avaliação da função cardíaca o método de Langerdorff, que consiste em isolar o coração e perfundir com solução Krebs-Henseleit (mM; $\mathrm{NaCl} 188$, $\mathrm{NaHCO}_{3}$ 25; Glicose 11,1; KCL 4,7; KH${ }_{2} \mathrm{PO} 41,2 ; \mathrm{MgSO}_{4} 7 \mathrm{H}_{2} \mathrm{O} 1,17 ; \mathrm{CaCl}_{2} 6 \mathrm{H}_{2} \mathrm{O}$ $2,5)$ sob gaseamento constante com a mistu$\mathrm{ra} \mathrm{O}_{2}: \mathrm{CO}_{2}(95: 5 \%)$, impelida por um sistema de pressão de perfusão conectado a uma cânula inserida na aorta (figura 3), segundo a proposta de Doring e Dehnert (1988). ${ }^{13}$

A pressão de perfusão foi de 60-70 $\mathrm{mmHg}$, que é adequada para ratos, para tanto, foi realizado o seguinte protocolo: 20 minutos de perfusão normal seguido de 60 minutos de solução de Krebs contendo noradrenalina $10^{-6} \mathrm{M}$ através de bomba de perfusão regulada em $2 \mathrm{~mL} \cdot \mathrm{min}^{-1} \cdot \mathrm{g}^{-1}$. A avaliação dos dados foi feita por meio da análise de variância, seguida do teste de Tukey, $\mathrm{p}<0,05$.

Para a avaliação estatística, os dados foram inicialmente submetidos ao teste de normalidade (Kolmogorov-Smirnov), e sequencialmente à análise de variância (ANOVA), seguida de post test Tukey com significância de 5\%.

\section{Resultados}

$\mathrm{Na}$ avaliação do peso dos animais foram comparados os grupos controle (C) e escolióticos (E), sendo verificado que os ratos escolióticos (E) apresentaram o peso $30 \%$ menor se comparado ao controle $(393,32 \pm 10 \mathrm{~g}$

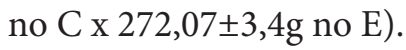

A seguir, foi avaliada a frequência cardíaca (batimento por minuto, bpm) e não foi observado diferença significativa entre os grupos $(151 \pm 30 \mathrm{bpm}$ no C X 169.6 $\pm 22 \mathrm{bpm}$ no E). Dentro do perfil eletrocardiográfico, foi calculado o ângulo SÂQRS e foram ve-

Figura 2 - Obtenção do registro eletrocardiográfico. A) Aparelhagem utilizada para a realização do registro; B) Posicionamento dos eletrodos no corpo do animal.
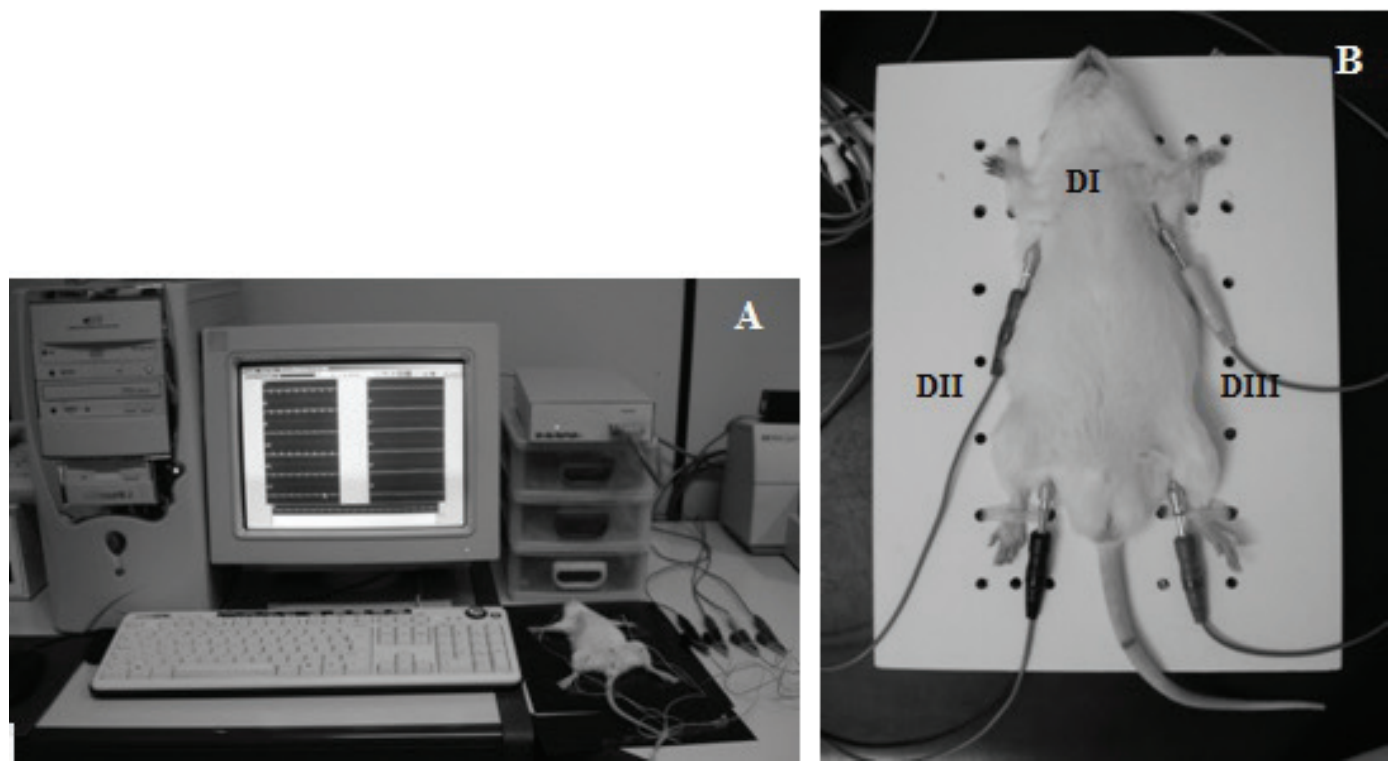
rificados valores médios de $88 \pm 3^{\circ}$ no grupo controle e $99 \pm 2^{\circ}$ no grupo escoliótico, indicando um desvio de $11^{\circ}$ em decorrência da escoliose (figura 4).

A análise dos parâmetros eletrocardiográficos mostrou que nem a duração da onda $\mathrm{R}$ nem o intervalo PR diferem entre os grupos, de maneira que a duração corresponde a $34,4 \pm 7,5 \mathrm{~ms}$ no grupo controle, e 29,2 $\pm 2.0 \mathrm{~ms}$ no grupo escoliótico. No mesmo contexto de análise, foi verificado que o intervalo PR apresentou valores de $42 \pm 3.0 \mathrm{~ms}$ no grupo controle, e $45 \pm 4.0 \mathrm{~ms}$ no grupo escoliótico.

A seguir, o estudo foi direcionado à avaliação do tempo do intervalo QRS (ms) sendo observado $59 \pm 7,4 \mathrm{~ms}$ no grupo controle e $69 \pm 1.1 \mathrm{~ms}$ no grupo escoliótico, indican- do que não houve alteração nesse padrão. O mesmo perfil também foi verificado no intervalo QTc (que é o intervalo QT corrigido pela fórmula de Bazett) onde não houve diferença entre os grupos sendo representado por $203 \pm 6.5 \mathrm{~ms}$ no grupo controle e $216 \pm 5,5 \mathrm{~ms}$ no grupo escoliótico.

Após a constatação da ausência de alterações no perfil de condução dos sinais elétricos cardíacos, aplicou-se o método de Langendorff, para avaliar a pressão arterial e a força de contração na presença de noradrenalina. Nesse sentido, pode ser observado na figura 5 que o grupo controle apresentou elevação de $97 \%$ na presença de noradrenalina, enquanto o grupo escoliótico mostrou elevação de $76 \%$, porém sem diferença estatisticamente significativa.

Figura 3 - Metodologia de avaliação da atividade de coração isolado e perfundido com solução fisiológica, sob pressão constante através de uma cânula inserida no ventrículo esquerdo, de acordo com Langendorff.

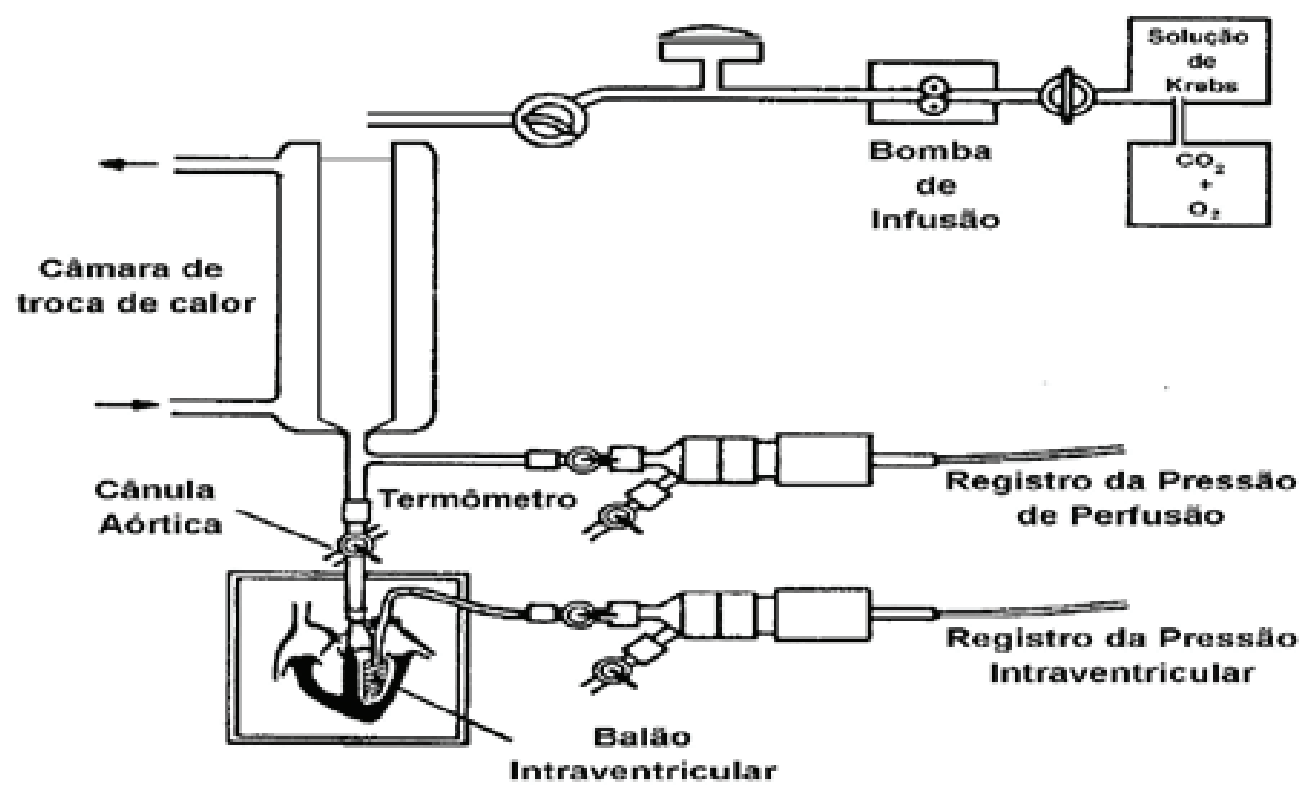


Por fim, foi avaliado o padrão $\mathrm{dP} / \mathrm{dt}+$, que indica as relações máximas de aumento na pressão sistólica e foi observado que o grupo controle não diferiu do escoliótico, uma vez que o controle apresentou elevação de 48,7\%, enquanto no grupo escoliótico a elevação foi de 47,3\% com a introdução da noradrenalina no meio de perfusão (figura 4).

Figura 4 - Posição do coração em ratos controle (A) e escolióticos (B). A seta mostra o eixo médio do coração.

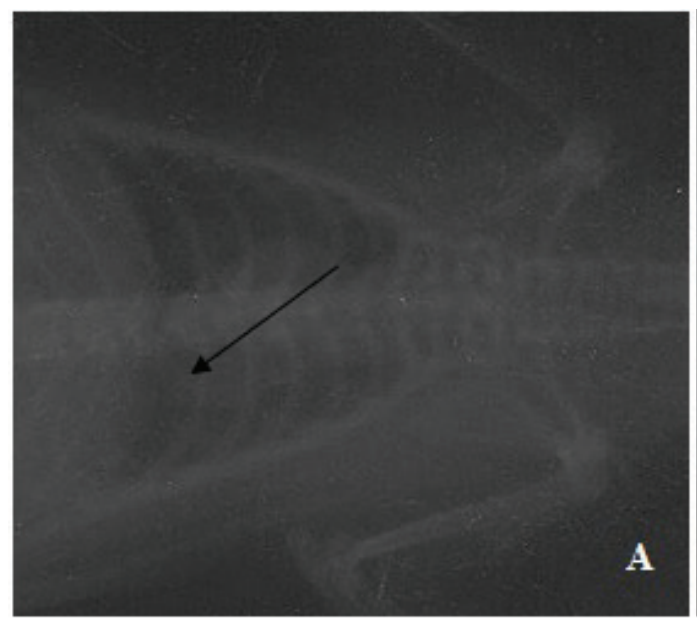

\section{DISCUSSÃ̃o}

Entre as diferentes nuanças de avaliação de funções do sistema cardiovascular há um grande interesse na busca de entender as relações entre deformidades de coluna e alterações nas dinâmicas que regem a atividade cardíaca. Na década de 80 , houve um importante estudo que avaliou o perfil cardiovascular de 802 humanos escolióticos e não encontrou diferença entre diferentes parâmetros das ondas eletrocardiográficas. ${ }^{14}$ Nesse período, também foi realizada uma avaliação eletrocardiográfica em pacientes com escoliose severa e com cifose e não foi verificado alteração significativa, fato que sugere que mudanças eletrocardiográficas não têm correlação com as deformidades cifóticas ou escolióticas. ${ }^{15}$

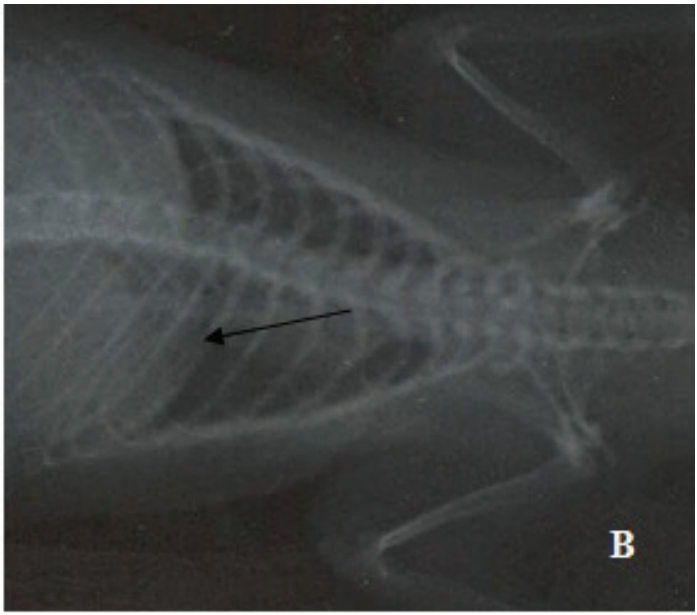

Há estudos que sugerem que desordens cardiovasculares frequentemente são detectadas em pacientes escolióticos, no entanto, essas alterações sempre acompanham síndromes, por exemplo, a síndrome de Marfan's, ataxia de Friedreich's, fatos que sugerem forte relação gênica. ${ }^{16}$ Essa sugestão foi reiterada no estudo de Choudhry et al. (1988), ${ }^{17}$ onde foi verificada, em pacientes escolióticos, a presença de potenciais indutores de artefatos eletrocardiográficos representados por taquicardia supraventriculares, no entanto, esse padrão ocorreu somente em crianças com a síndrome de Goldenhar.

Mais recentemente, Durmala et al. $(2006)^{18}$ avaliaram 90 crianças portadoras de escoliose, com idade entre 7 anos a 18 anos, e observaram que $44 \%$ dos pacientes apresentavam elevação no tempo do inter- 
Figura 5 - Pressão arterial ( $\mathrm{mm} / \mathrm{Hg})$ e força de contração cardíaca determinada pelo método de Langendorff. Em (A), pode-se verificar a Pressão Sistólica Ventricular e em (B) a dP/ $\mathrm{dt}+$ que indica as relações máximas de aumento na pressão sistólica de ratos controle ( $\square$ ) e escolióticos $(\diamond)$. A noradrenalina $10^{-6}$ foi administrada no tempo zero (T0). Os valores correspondem à média \pm epm, $\mathrm{n}=8$.
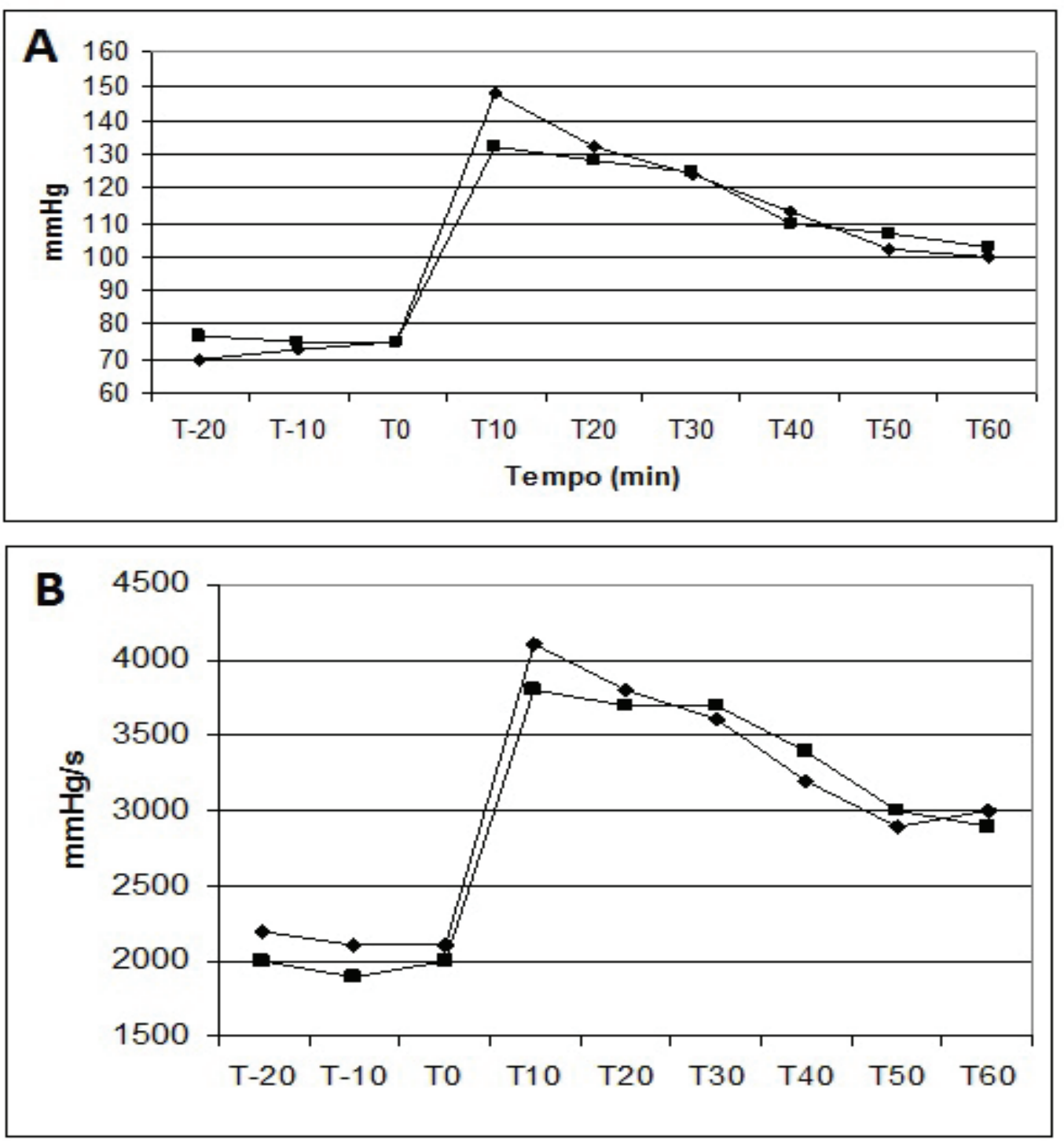
valo QRS, nesse interim, os autores sugeriram que as alterações podem ter relações com desvios no eixo do coração induzido pela deformidade.

Stubgen $(2008)^{19}$ relatou anormalidades na condução de potenciais elétricos na câmara ventricular direita em humanos portadores de síndrome da rigidez da coluna, e sugeriu que essa alteração acompanha outros eventos como a taquiarritmia supraventricular em situações de hipoxia ou hipercapnia.

Com relação a modelos experimentais utilizados na indução da escoliose, não há registros de acompanhamento eletrocardiográfico concomitante à progressão da escoliose, o que torna este estudo pioneiro.

Com relação ao menor peso dos animais escolióticos, pode-se inferir que possa estar relacionado com redução na ingesta de ração, já que esta passou de $60 \pm 5.0 \mathrm{~g} / \mathrm{dia}$ no grupo controle para $45 \pm 3.0 \mathrm{~g} /$ dia no grupo escoliótico. Possivelmente, os mecanismos associados à imobilização provocada pelo processo de indução da escoliose promovam alterações no equilíbrio homeostático, com reflexo no controle neural das vísceras, devido à curvatura que apresenta ação compressiva na região abdominal e reflexamente promova modificação da ingesta, fato que pode acompanhar o processo de indução da escoliose, de acordo com a proposta de Brandão (1995). ${ }^{20}$

Ao comparar-se ratos controle com os escolióticos, tendo como base a amplitude e intensidade de propagação dos sinais elétricos nas diferentes áreas cardíacas, não foram verificadas mudanças na dinâmica cardiovascular representada tanto pelas ondas do ECG quanto pela força contrátil determinada pelo método de Langendorff. Um ponto merecedor de destaque é que recentemente demonstramos que o modelo também não promoveu alteração comportamental. ${ }^{21}$

\section{CONSIDERAÇões FINAIS}

O modelo de indução de escoliose por meio de coletes de PVC apresentou eficácia na indução da curvatura escoliótica e a deformidade estrutural não promoveu alterações no padrão de distribuição do sinal elétrico cardíaco, bem como, não promoveu modificação na dinâmica do controle da pressão arterial.

\section{REFERÊNCIAS}

1. Guimarães, JI, Nicolau JC, Polanczyk CA, Pastore CA, Pinho JA, Bacellar MSC et al. Diretriz de interpretação de eletrocardiograma de repouso. Arq. Bras. Cardiol. 2003; 80(2): 1-18.

2. Feldman J., Goldwasser GP. Eletrocardiograma: recomendações para a sua interpretação. Rev. SOCERJ. 2004; 17(4): 251-256.

3. Guyton AC. Tratado de Fisiologia Médica, 9. ed. Rio de Janeiro: Guanabara Koogan; 2003, 108-118.

4. Dickson RA. Leatherman, KD. The management of spinal deformities. London: Wrigth. 1988. 1-54.

5. Byrd III JA. Current theories on the etiology of idiopathic scoliosis. Clin Orthop. 1988; 229: 114-119. 
6. Coillard C., Rivard CH. Vertebral deformities and scoliosis. Eur Spine J. 1996; 5: 91-100.

7. Dabney KW, Salzman SK, Wakabayayashi T. Experimental scoliosis in the rat. Spíne, 1988; 13(5): 472-477.

8. Joe T. Studies of experimental scoliosis produced by electric stimulation. Nippon Zasshi, 1990; 57(5): 416-426.

9. O'kelly C., Wang, X., Raso, J., Moreau, M., Mahood, J., Zhao, J. The production of scoliosis after pinealectomy in young chickens, rats and hamsters. Spine. 1999; 1(24): 35-43.

10. Stokes IA, Gwadera J., Dimock A., Aronsson DD. Mechanical modulation of vertebral and tibial growth: diurnal versus full-time loading. Study Health Technol Inform. 2002; 91: 97-100.

11. Silva CA, Guirro RRJ, Delfino GB, Arruda EJ. Proposal of non-invasive experimental model to induce scoliosis in rats Proposta de modelo experimental não-invasivo para indução de escoliose em ratos. Rev Bras Fisioter, 2012, 16 (3), 254-260.

12. Kumar S., Kela AK, Mehta VL, Shukla AK. Preferred anesthetic agents in experimental cardiology: A study on rat electrocardiogram. Indian J. Pharmacol. 1995; 27: 127-129.

13. Doring $H J$, Dehnert $H$. The isolated perfused warm-blooded heart according to LANGENDORFF. Est Germany. 1988: Biomesstechnik- Verlag March GmbH, D7806.

14. Hwang B., Simon G., Keim HA, Krongrad E. The electrocardiogram in patients with scoliosis. J. Electrocardiol. 1982; 15 (2): 131-136.

15. Peters S. Electrocardiography changes in scoliosis and kyphosis deformities of the thorax. Z. Orthop Ihre Grenzgeb. 1987; 125 (1): 14-18.

16. Godfrey S. Respiratory and cardiovascular consequences of scoliosis. Respiration. 1970; 27(suppl. 1): 67-7.

17. Choudhry, DK, Stayer, AS, Rehman, MA, Schwartz, RE. Electrocardiographic artifact with SSEP monitoring unit during scoliosis surgery. Paediatr Anaesth. 1998; 8(4): 341-343.

18. Durmala J., Sosnowska M., Sosnowski, M. Prevalence of subtle cardiac electrical abnormalities on children with idiopathic scoliosis. Stud Health Technol Inform. 2006; 123: 425-430.

19. Stubgen JP. Rigid Spine Syndrome: A Noninvasive Cardiac Evaluation. Pediatric Cardiology, 2008, 29: 45-49.

20. Brandão ML. Psicofisiologia. Rio de Janeiro: Atheneu; 1995. 42-54.

21. Silva, CA, Guirro, RRJ, Fonseca, W, Arruda, EJ, Grassi, DO. assessment of rat behavior with induced scoliosis by polyvinyl chloride vests. Journal Chinese Clinical Medicine. 2008; 3(11): 621-626.

\section{DAdos dos Autores}

\section{Carlos Alberto da Silva}

Doutor em Ciências Fisiológicas pela Universidade Estadual de Campinas. Professor no Programa de Pós-Graduação em Ciências do Movimento Humano na Universidade Metodista de Piracicaba. Piracicaba/SP - Brasil. casilva@unimep.br

\section{Vitor Alexandre Pezolato}

Doutorando de Fisioterapia na Universidade Federal de São Carlos. São Carlos/SP - Brasil. vitor. pezolato@gmail.com

\section{RODRIgo Dias}

Doutorando em Ciências do Movimento Humano na Universidade Metodista de Piracicaba. Piracicaba/SP - Brasil.rdiasd@gmail.com 


\section{Alexandre Reis Taveira de Souza}

Mestre em Biologia Funcional e Molecular pela Universidade Estadual de Campinas. Professor de Biologia para o ensino médio, curso pré-vestibular e Professor Adjunto I da Universidade Paulista. Campinas/SP - Brasil. profalexandre2@gmail.com

\section{Bruno Ferreira Gonçalves e Silva}

Graduando do curso de Medicina Veterinária pela Universidade Paulista. Campinas/SP - Brasil. bruninho0201@hotmail.com

Submetido em: 22-10-2013

Aceito em: 5-4-2017 